\title{
Sea Ice Classification Using Multi-Frequency Polarimetric SAR Data
}

\author{
B. Scheuchl ${ }^{1}$, I. Hajnsek ${ }^{2}$, and I. Cumming ${ }^{1}$ \\ ${ }^{1}$ Dept. of Electrical and Computer Engineering, University of British Columbia, 2356 Main Mall, Vancouver, B.C., Canada \\ V6T1Z4 E-mail: bernds@ece.ubc.ca, ianc@ece.ubc.ca \\ ${ }^{2}$ German Aerospace Center, Institute for Radio Frequency Technology and Radar Systems, Department SAR Technology, \\ P.O. Box 1116, 82234 Wessling, Germany E-mail: irena.hajnsek@dlr.de
}

\begin{abstract}
This paper discusses the capability of the complex Wishart classifier for sea ice and classification using multifrequency, fully polarimetric SAR data. C-, L-, and P-band data acquired by the JPL AIRSAR in the Beaufort sea was used. Classification using the unsupervised Wishart classifier is a twostage process. An initial classification is required to seed the algorithm and can be derived using other classification methods. The Wishart classifier then used in iterations where the class means are updated after every step. The convergence of this approach is investigated. The Wishart classifier was found to be extremely dominant so that the classification result after a few iterations depends not necessarily on the initial classification used to derive the first class means. Even an initial classification derived with a random number generator leads to a good result after a few iterations.
\end{abstract}

\section{INTRODUCTION}

Sea ice is a challenging remote sensing target because of its complexity and temporal-variability. Operational sea ice monitoring is undertaken by several countries with coastlines affected by seasonal sea ice cover. Knowledge of the extent of the ice cover, as well as the ice types, present is of scientific as well as economic value. Spaceborne Synthetic Aperture Radar (SAR) sensors are a vital information source for ice centers around the world. Airborne SAR sensors do not provide the same coverage; however, several airborne SAR's are capable of acquiring multi frequency, fully polarimetric data. This provides much more complete information about the target. In this paper we investigate the potential of the complex Wishart classifier for unsupervised sea ice classification when multi-frequency data is available.

\section{Description Of The Test DAta}

In March 1988 NASA/JPL conducted an experiment in the Beaufort Sea involving remotely sensed data from various sensors and simultaneously collected meteorological data as well as measured ice profiles are available.

The airborne JPL AIRSAR system acquired SAR data over the chosen test site in a fully polarimetric mode in three frequencies (C-, L- and P-band). These complete data over sea ice were subject to intensive research and numerous publications reported on the analyses $[1,2,3]$.

This work was partly funded by MacDonald Dettwiler and Associates under contract to the Canadian Space Agency (No. 9F028-0-4902/06) as part of the Earth Observation Applications Development Program.
The scene used for the investigation discussed in this paper (scene 1372) was acquired on March 11, 1988 with scene center coordinates of $73.048^{\circ} \mathrm{N}$ and $142.285^{\circ} \mathrm{W}$ and incidence angle variation from 27 to 52 degrees. Figure 1 shows a false color composite of the three frequencies presented as total power images. Interpreting the color of the sea ice scene represents a visual classification: red floes are multiyear ice (MYI), they are surrounded by compressed first year ice (FYI) presented with a white color; dark blue areas represent smooth FYI; light blue lines are ridged FYI and rubble and black areas are newly forming ice in recently developed leads, according to a classification result in [3]. A detailed investigation on various polarimetric parameters can be found in [3].

\section{THE WISHART CLASSIFIER}

\section{A. Minimum Distance Classification}

The Wishart classifier is a Bayesian minimum distance classifier based on the probability distribution of the coherency matrix $T$ [4]. A pixel is assigned to the class to which a metric is minimum. In principal it is a supervised algorithm that requires a training data set. The use of the coherency matrix for classification ensures that the entire polarimetric information is utilized. However, the classifier works very much on statistical principles, that is relating the coherency matrix to a class mean, rather than exploiting the information on the physical scattering involved on the ground.

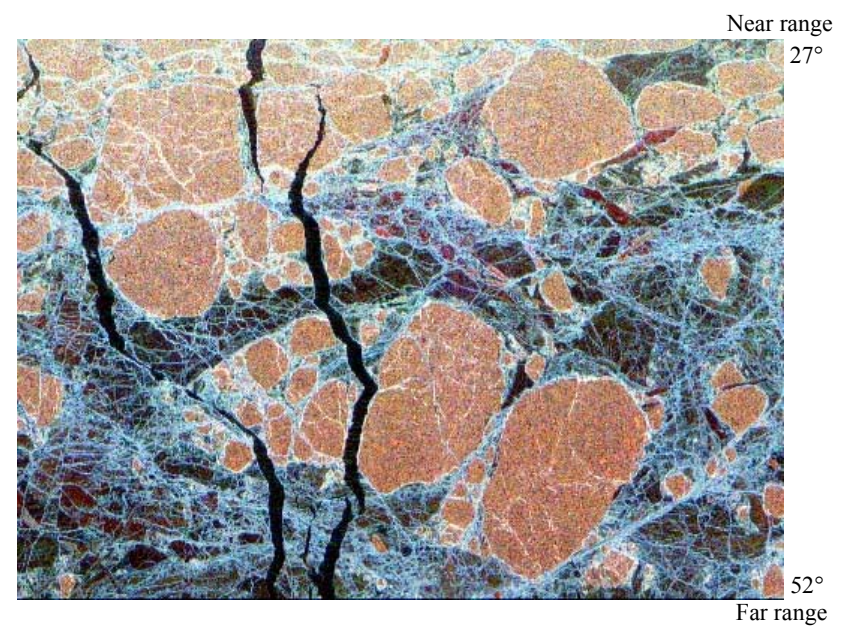

Fig. 1: RGB image using the total power (in $\mathrm{dB}$ ) of the three frequencies (red $=\mathrm{C}-$ band, green $=\mathrm{L}$ band, blue $=\mathrm{P}-\mathrm{band}$.) 
An investigation into how the physical scattering approach can be used in combination with the Wishart classifier is documented in [5].

The algorithm can be improved in two steps:

Firstly, the algorithm can be used iteratively. Here, the result of one iteration is used to generate the class means of the next step. One possible way of controlling the number of iterations is to monitor the number of pixels changing class during an iteration. If this number falls below a certain threshold, the iterations can be stopped. Or a fixed number of iterations can be used.

The second step to improve the capability of the algorithm is to use as initial input of the classifier the entropy/anisotropy/ $\alpha$-angle $(\mathrm{H} / \mathrm{A} / \alpha-$ angle) classification scheme to initialize the classes as proposed from [4], which provides classes relating to the underlying physical scattering mechanism. The classification result is unsupervised thus making the combined method unsupervised, too. The H/A/ $\alpha-$ angle classifier based on eigenvector analysis of the coherency matrix has been developed for land applications and is mostly used in combination with the Wishart classifier. Recently this combined unsupervised method was successfully used for Cband sea ice classification [6].

\section{B. Extension to multi-frequency polarimetric data}

An extension of the method for multi-frequency data is possible by using one of the following proposed metrics:

In the first approach, the single frequency concept is used to calculate three distances for a pixel to a class mean, one for each frequency. The distances are than summed up and the pixel is assigned to the class to which the combined distance has a minimum [7].

The second method combines all three $3 \times 3$ coherency matrices in one 9x9 matrix where the main diagonals of the small matrices form the main diagonal of the new matrix. This new matrix is then used to calculate the distance of a pixel to the various class means [4].

If the frequencies are well separated, no correlation between different frequencies is present and both results are equal. The 9x9 matrix approach allows to account for inter frequency correlation which is not the case in the three matrix approach.

\section{Extension to multi-frequency polarimetric data}

One initial classification used to generate the first class means was produced with a random number generator. Each pixel of the scene was randomly assigned a number between one and six representing classes with the distribution of the numbers being equal. Based on this pseudo classification the initial class means were generated.
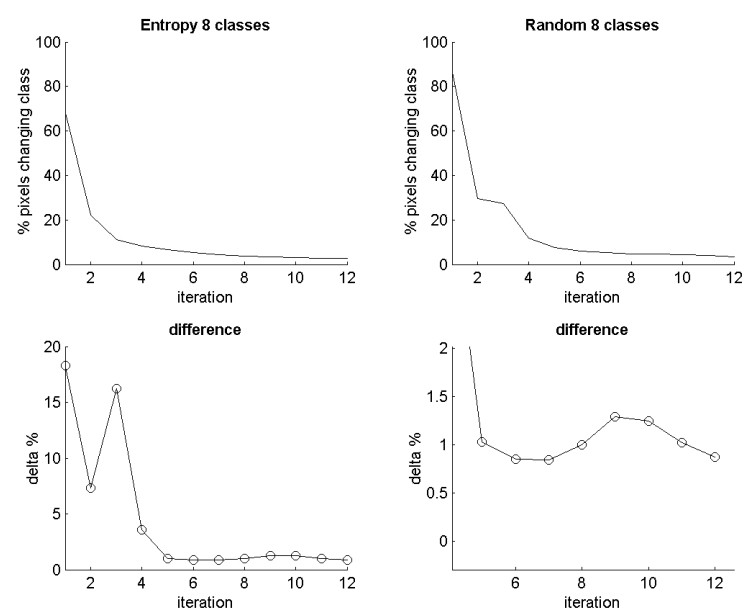

Fig. 2: Comparison of the two methods proposed for multi frequency Wishart classification. Pixels changing classes per iteration are monitored a) entropy based startsequence, b) random start sequence, c) b-a, d) c zoomed

A random start sequence shows the robustness of the iterative approach. The convergence of the algorithm would probably be better if another initial classification was used that actually represents a classification of the scene. Also, the result could then be reproduced. Figure 2 shows a comparison between a random number start sequence and an entropy based start sequence where eight classes are derived by thresholding each entropy image at 0.5 . While there is indeed an improvement in the convergence of the algorithm, the difference is not as drastic as one might have expected. A significant difference in the number of pixels changing class can only be reported for the first four iterations. After five iterations the difference is about $1 \%$ with the entropy based start sequence approach showing less change.

The confusion matrix in Table I shows that the results are indeed very similar once the classes are assigned to ice types. All ice types were chosen in acordence to previously published results.

The automatically generated classes were assigned colors and are shown in Figures 3 and 4. The color code and the class interpretation for both images is given in Table II. Differences in the appearance of the two images are the result of classes belonging to the same ice type showing in different proportions.

TABLE I

Confusion Matrix For Random Vs Entropy Based Start Classification

\begin{tabular}{crrrr}
\hline & ThI / SFYI & \multicolumn{1}{c}{ RFYI / R } & \multicolumn{1}{c}{ CFYI } & \multicolumn{1}{c}{ MYI } \\
\hline ThI / SFYI & $100.00 \%$ & $1.80 \%$ & $0.00 \%$ & $0.01 \%$ \\
RFYI / R & $0.00 \%$ & $97.47 \%$ & $30.25 \%$ & $0.23 \%$ \\
CFYI & $0.00 \%$ & $0.00 \%$ & $69.75 \%$ & $2.18 \%$ \\
MYI & $0.00 \%$ & $0.73 \%$ & $0.00 \%$ & $97.58 \%$ \\
\hline
\end{tabular}




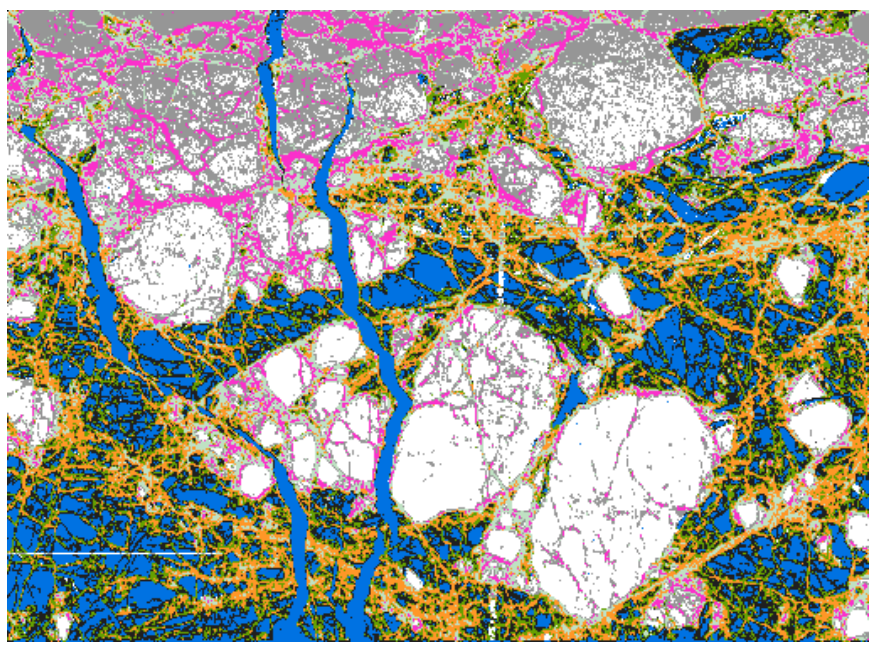

Fig. 3. Classification result after 12 iterations using an entropy based classification with eight classes as initial classification.

\section{CONCLUSIONS}

The Wishart classifier is a useful tool for sea ice classification and can be easily extended to handle multi-frequency data. The algorithm is so dominant that it depends very little on the initial classification used. A start sequence generated with a random number generator leads to a useful result. An initial classification that is derived from the data shows a slightly better performance with respect to convergence of the algorithm. It also provides the possibility to reproduce the classification result. For the three frequency case an entropy based start sequence is suggested.

\section{ACKNOWLEDGMENTS}

The authors thank Ron Caves from MacDonald Dettwilerand Gordon Staples from RADARSAT International for their valuable comments as well as NASA/JPL for providing the airborne SAR data and the ground measurements.

TABLE II

Class Assignment To Ice Types

\begin{tabular}{ccc}
\hline Class & Colors & Description \\
\hline ThI / SFYI & blue & $\begin{array}{c}\text { New forming thin ice / } \\
\text { Smooth first year ice }\end{array}$ \\
\hline RFYI / R & $\begin{array}{c}\text { orange } \\
\text { green } \\
\text { black }\end{array}$ & $\begin{array}{c}\text { Ridged first year ice / } \\
\text { Rubble }\end{array}$ \\
\hline CFYI & pink & Compressed First Year Ice \\
& pastell green & Multi year Ice \\
\hline MYI & white & \\
& gray &
\end{tabular}

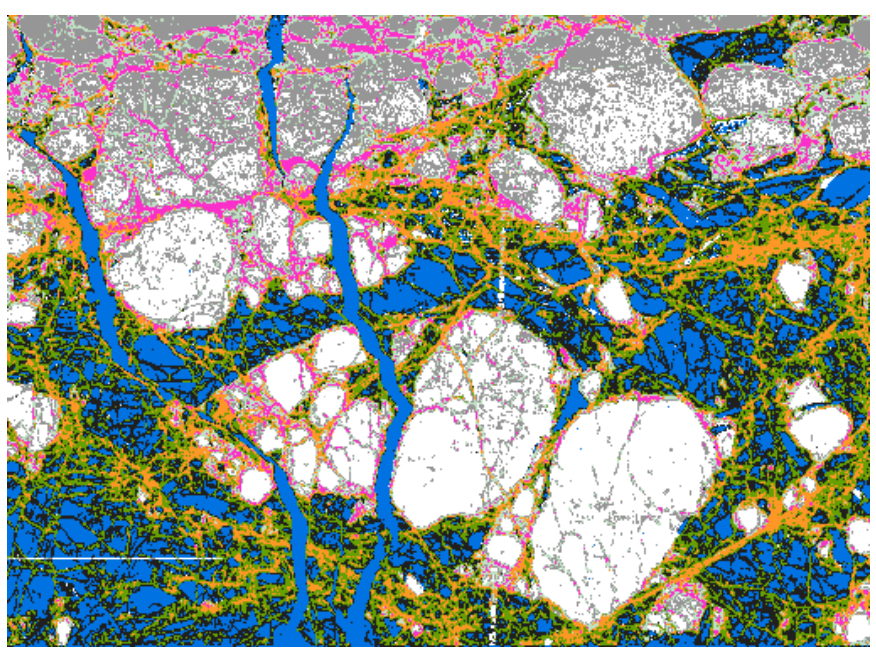

Fig. 4. Classification result after 12 iterations using start classification generated with a randomn number generator producing eight evenly distributed classes as initial classification.

\section{REFERENCES}

[1] S.V. Nghiem and C. Bertola, "Study of Multi Polarization Cband backscatter Signatures for Arctic Sea Ice Mapping with Future Satellite SAR," Canadian Journal of Remote Sensing, vol. 27, no. 5, pp. 387-402, October 2001.

[2] M.R. Drinkwater, R. Kwok, D.P. Winebrenner, and E. Rignot, "Multifrequency Polarimetric Synthetic Aperture Radar Observations of Sea Ice," Journal of Geophysical Research, vol. 96, no. C11, pp.20679-20698, November 1991.

[3] E. Rignot, and M.R. Drinkwater, "Winter sea-ice mapping from multi-parameter synthetic-aperture radar data," Journal of Glaciology, vol. 40, no. 136, pp. 31-35, 1994.

[4] J.-S. Lee, M. Grunes and R. Kwok, "Classification of multilook polarimetric SAR imagery based on complex Wishart distribution," Int. J. Remote Sensing, vol. 15, no. 11, pp. 22992311, 1994.

[5] B. Scheuchl, I. Hajnsek, and I. Cumming, " Model Based Sea Ice Classification Using Polarimetric SAR Data," in Proc. IGARSS'02, 2002 (these proceedings).

[6] B. Scheuchl, R. Caves, I. Cumming, and G. Staples, "Automated Sea Ice Classification Using Spaceborne Polarimetric SAR Data," in Proc. IGARSS, 2001.

[7] L. Ferro-Famil, E. Pottier, and J.S. Lee, "Unsupervised Classification of Multifrequency and Fully Polarimetric SAR Images Based on the H/A/Alpha-Wishart Classifier," IEEE Trans. Geosci. Remote Sensing, vol. 39, no. 11, pp. 2332-2342, November 2001. 